Check for updates

Cite this: Phys. Chem. Chem. Phys., 2018, 20, 11412

Received 14th February 2018, Accepted 23rd March 2018

DOI: $10.1039 / c 8 c p 01058 g$

rsc.li/pccp

\section{Tailored photocleavable peptides: fragmentation and neutralization pathways in high vacuum $\dagger$}

\author{
M. Debiossac, (D) $\ddagger^{a}$ J. Schätti, $\ddagger^{\mathrm{b}}$ M. Kriegleder, ${ }^{\mathrm{a}}$ P. Geyer, ${ }^{\mathrm{a}}$ A. Shayeghi, (D) a \\ M. Mayor, (iD bcd M. Arndt (D)*a and V. Köhler (DD *b
}

\begin{abstract}
Photocleavable tags (PCTs) have the potential for excellent spatio-temporal control over the release of subunits of complex molecules. Here, we show that electrosprayed oligopeptides, functionalized by a tailored ortho-nitroarylether can undergo site-specific photo-activated cleavage under UV irradiation (266 nm) in high vacuum. The comparison of UV photodissociation (UVPD) and collision-induced dissociation (CID) points to the thermal nature of the cleavage mechanism, a picture corroborated by the temperature dependence of the process. Two competing photodissociation pathways can be identified. In one case a phenolate anion is separated from a neutral zwitterion. In the other case a neutral phenol derivative leaves a negatively charged peptide behind. To understand the factors favoring one channel over the other, we investigate the influence of the peptide length, the nature of the phenolic group and the position of the nitro-group (ortho vs. para). The observed gas phase cleavage of a para-nitro benzylic ether markedly differs from the established behavior in solution.
\end{abstract}

\section{Introduction}

The charge state of peptides and proteins affects their chemical and biological behavior through intermolecular electrostatic interactions as well as by modulation of their geometry and folding, electronic and vibrational energy structure, ${ }^{1}$ and their electro-optical or collisional ${ }^{2,3}$ properties. Spectroscopic studies of biomolecules in the gas phase are interesting as they specifically allow identifying the role of matrix effects. ${ }^{4-6}$

The combination of both aspects, i.e. charge control of biomolecules in the gas phase is relevant for molecular trapping, ${ }^{7,8}$ optical $^{9}$ and photo-electron spectroscopy, ${ }^{10,11}$ as well as for electron or femto-second X-ray diffraction. ${ }^{12,13}$ Several methods for charge manipulation have been studied in the past, such as atomic collisions, ${ }^{14}$ chemical reactions, ${ }^{15}$ and low-energy electron attachment. $^{16}$

Laser-induced processes are intriguing since they are compatible with ultra-high vacuum requirements, can achieve high

\footnotetext{
${ }^{a}$ Faculty of Physics, University of Vienna, VCQ, Boltzmanngasse 5, A-1090 Vienna, Austria.E-mail: markus.arndt@univie.ac.at

${ }^{b}$ Department of Chemistry, University of Basel, Mattenstrasse 24a, BPR 1096, CH-4058 Basel, Switzerland. E-mail: valentin.koehler@unibas.ch ${ }^{c}$ Institute of Nanotechnology (INT), Karlsruhe Institute of Technology (KIT), Hermann-von-Helmholtz-Platz 1, D-76344 Eggenstein-Leopoldshafen, Germany

${ }^{d}$ Lehn Institute of Functional Materials (LIFM), Sun Yat-Sen University (SYSU), Xingang Rd. W., Guangzhou, China

$\dagger$ Electronic supplementary information (ESI) available: Details concerning the chemical synthesis and NMR characterization of the molecules, the data analysis, computational details and additional discussions. See DOI: $10.1039 / \mathrm{c} 8 \mathrm{cp} 01058 \mathrm{~g}$ ¥ These authors contributed equally to this work.
}

efficiency and combine high spatial resolution with subnanosecond timing. UV electron photodetachment (ED) has recently been successfully demonstrated on insulin polyanions, ${ }^{17}$ however, in complex molecules it competes with photodissociation (PD). ${ }^{18}$

It has been shown that photocleavage can be optimized using tailored tag molecules that respond to UV light ${ }^{19,20}$ and visible light, ${ }^{21}$ also for peptides with ionization energies exceeding the photon energies of table-top lasers. ${ }^{22}$ The heterolytic removal of the leaving group (LG) from a singly charged photo-tagged peptide anion is a promising strategy for the controlled generation of neutral zwitterions in the gas phase ${ }^{23}$ (Scheme 1) and can be relevant for proteomics. ${ }^{24-26}$

Our work aims at developing tools that enable the generation of continuous beams of neutral, slow and internally cold peptides and proteins for matter-wave interferometry. ${ }^{27}$ Such controlled beams are valuable for fundamental tests of quantum physics, enable new measurements of molecular electronic, ${ }^{28}$ optical and magnetic properties, ${ }^{29,30}$ as well as optical and infrared spectroscopy under controlled interaction-free conditions. ${ }^{31}$

Here, we study tailored oligopeptides with a photocleavable tag in an electrospray mass spectrometer, ${ }^{32}$ aiming at the controlled charge removal from singly charged anions by photodissociation (PD) at a tailored cleavage point. ${ }^{33}$ For that purpose, we have synthesized non-aromatic oligopeptides containing between three and twelve amino acids (1-4), and a covalently attached photocleavable tag (PCT) with a leaving group that is supposed to split off upon absorption of one or several UV photons (Scheme 1 and ESI $\dagger$ ). We have synthesized the four different LGs (a-d) to 


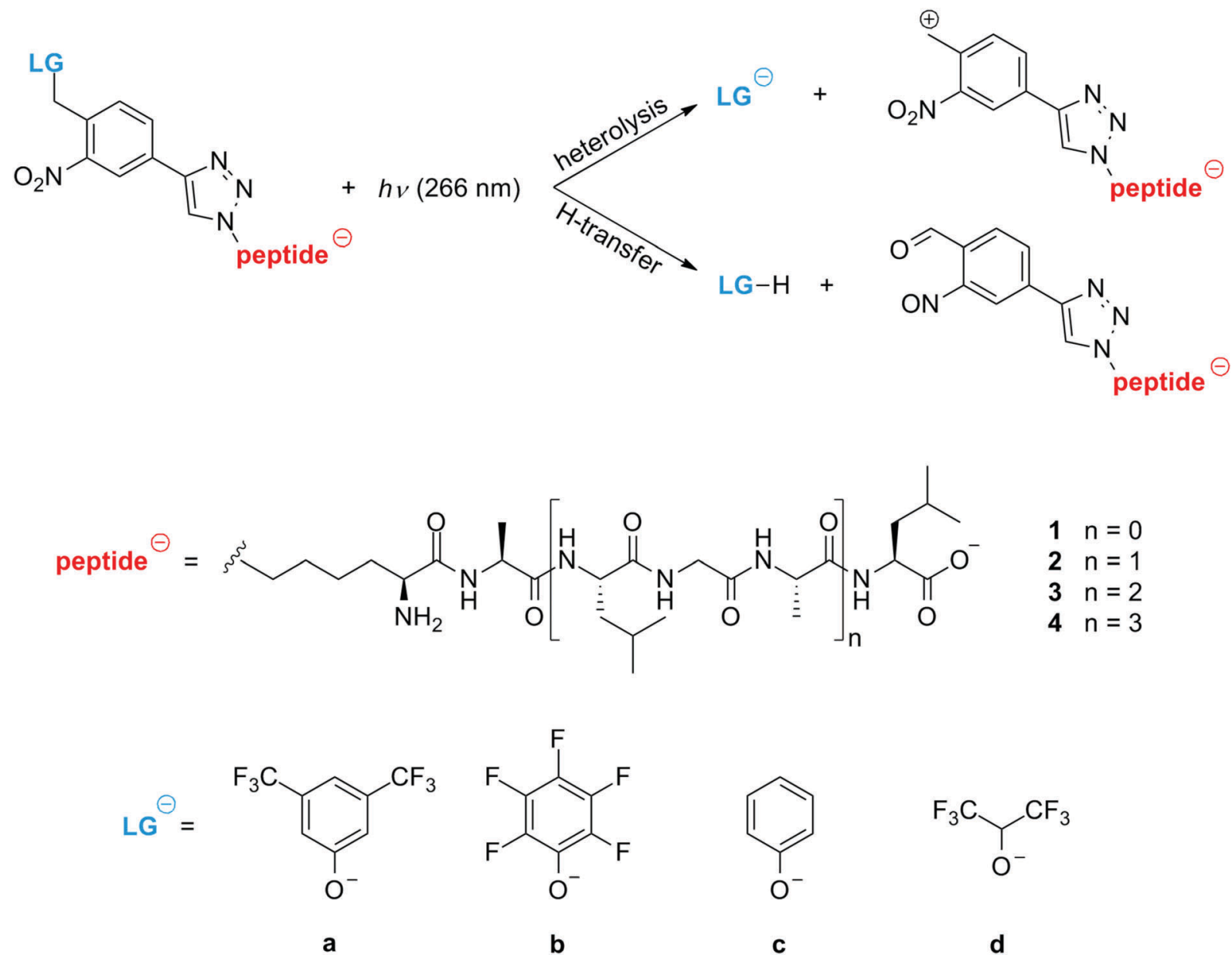

Scheme 1 Photocleavable tags (PCT), reaction scheme, oligopeptides and leaving groups (LG) used in this study. Upon irradiation with $266 \mathrm{~nm}$ UV light the functionalized peptides can undergo either heterolytic cleavage or dissociation with simultaneous proton transfer. The functionalized peptides differ in their amino acid sequence Lys-Ala-(Leu-Gly-Ala) ${ }_{n}$-Leu and in their leaving group (LG) 1a, $1 \mathbf{b}, \mathbf{1} \mathbf{c}$, 1d. The index $n=0-3$ labels the oligopeptides from a tripeptide $\mathbf{1 a}$ to the dodecapeptide $\mathbf{4 a}$.

investigate their influence on the cleaving efficiency and in all cases the aromatic PCT is designed to be the dominant UV absorber in the tagged peptide.

The experiments are performed using a customized ESI-QTOF mass spectrometer, as shown in Fig. 1. The electro-sprayed ions are guided into high vacuum by a stack of ring electrodes. They are mass-selected by a quadrupole ion filter, temperaturecontrolled by the buffer-gas in the first hexapole ion guide (marked in blue in Fig. 1), photo-activated by UV laser light inside the second hexapole ion guide (without buffer gas, marked in red) and detected using a time-of-flight mass spectrometer. A pulsetube cooler was fitted to the first hexapole, allowing to set a temperature of between $T=60-300 \mathrm{~K}$. Pulsed ultraviolet laser light ( $\lambda=266 \mathrm{~nm}, 10 \mathrm{~ns},<1 \mathrm{~mJ}$ per pulse) was aligned to be collinear and counter-propagating to the ion beam.

\section{Results and discussion}

Fig. 2a and b show the UVPD (a) and CID (b) mass spectra of the tripeptide 1a. The fragment at $229 \mathrm{u} / \mathrm{e}$ results from heterolytic cleavage of the leaving group a. Both mass spectra show the desired LG-anion a as the only fragment, suggesting that UVPD and CID follow a similar mechanism.

Fig. $2 \mathrm{c}$ and $\mathrm{d}$ trace the UV photodepletion efficiency for the tripeptide 1a as a function of the laser fluence and for two different molecular temperatures. The molecules interact with a buffer gas at $300 \mathrm{~K}$ (c) or $60 \mathrm{~K}$ (d) prior to the PD experiments. We define the UVPD efficiency as $1-S / S_{0}$. It measures the reduction of the parent ion signal in the presence $(S)$ or absence $\left(S_{0}\right)$ of the UV light. Its dependence on the laser fluence $F$ is derived from kinetic rate equations ${ }^{21,34}$

$$
1-S / S_{0}=1-\alpha+\alpha(1+\gamma \sigma F) \mathrm{e}^{-\sigma F}
$$

with $\alpha$ as the spatio-temporal overlap between the UV laser beam and the ion beam, $\gamma$ the fraction of two-photon processes and $\sigma$ the PD cross section as a lower bound to the absolute absorption cross section (see Fig. S1, ESI $\dagger$ ). The temperature of the buffer gas determines whether one $(\gamma=0)$ or at least two photons $(\gamma=1)$ are needed to deplete the parent ion signal. From Fig. 2a we extract $\alpha=0.4 \pm 0.1$ and $\sigma=0.4 \pm 0.1 \AA^{-2}$. 


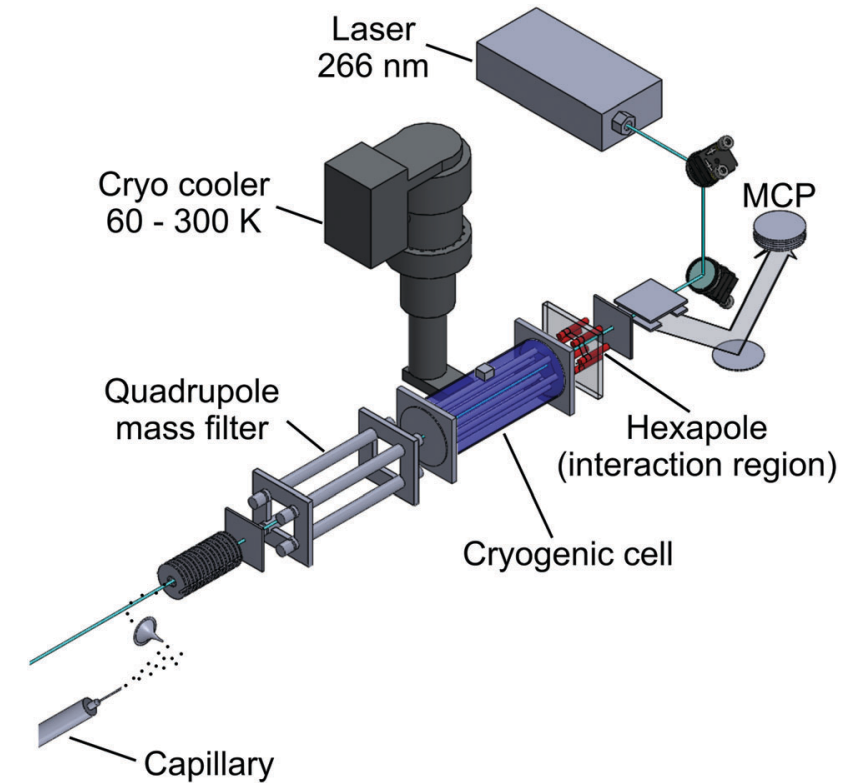

Fig. 1 Experimental setup. Ions are sprayed and mass-selected in a 2D quadrupole filter (MS1) and temperature controlled in the cryogenic hexapole ion guide before interacting with short (10 ns) $266 \mathrm{~nm}$ laser pulse inside the second hexapole guide.
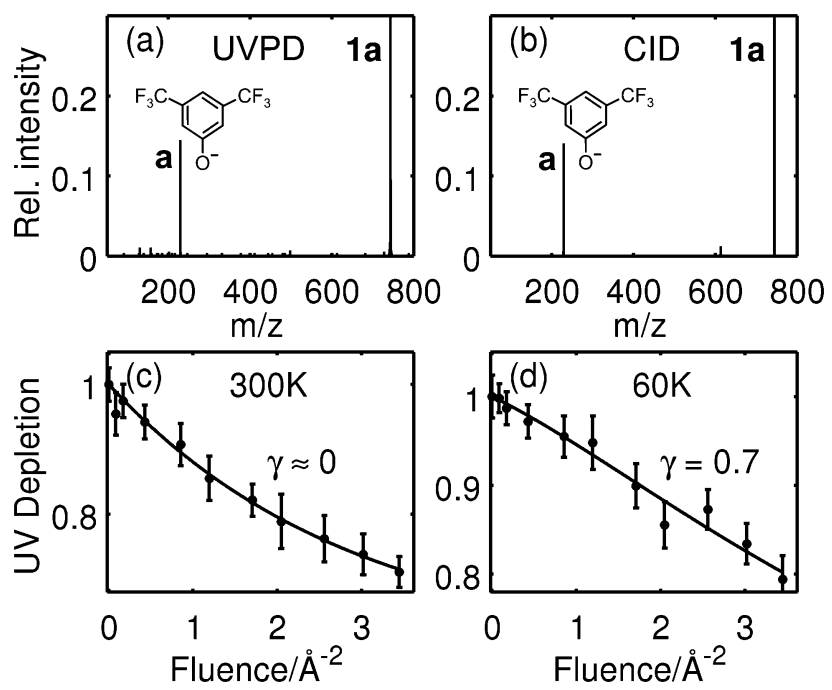

Fig. 2 ( $a$ and $b)$ The UVPD and CID mass spectra of the tripeptide 1a ( $m=744 \mathrm{u} / \mathrm{e}$ ) show one and the same fragment a at $m / z=229 \mathrm{u} / \mathrm{e}$. The CID spectrum was recorded at an ion energy of $28 \mathrm{eV}$ in collision with room temperature argon atoms. (c and d) Temperature dependence of the photodepletion efficiency: at a molecular temperature of $300 \mathrm{~K}$, the UVDP curve can be fitted by a pure exponential decay, corresponding to a singlephoton process (c). At $T \simeq 60 \mathrm{~K}$ a large fraction of molecules must absorb two or more photons before they fragment.

While single-photon cleavage prevails at $300 \mathrm{~K}$, the data are best fitted by a $70 \%$ probability for a two-photon process when the molecules are $60 \mathrm{~K}$ cold.

This suggests that the cleavage process depends on the molecular heat capacity, which increases with peptide length (Fig. 3). More photons are then required for heterolytic cleavage
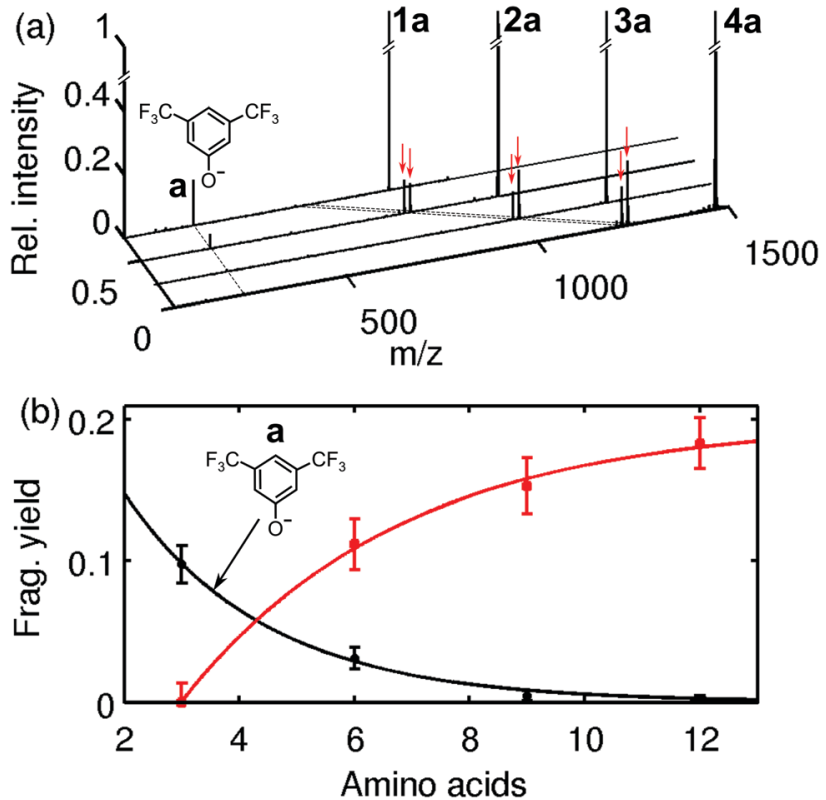

Fig. 3 (a) UVPD mass spectra for peptides $1 \mathbf{1}-\mathbf{4 a}$ for maximum laser fluence of $3.3 \AA^{-2}$. The fragment at 229 u/e results from heterolytic cleavage of the LG. Red arrows indicate the fragments formed due to proton transfer dissociation (M-LG-H and M-LG-H-16). (b) Fragment yield for $L G$ a (dark circles) and for fragments due to proton transfer dissociation (red squares) as a function of the peptide length. Points and error bars represent experimental values, full lines display curve fitting using the exponential depletion function.

to occur on the experimental time scale (Fig. S3, ESI $\dagger$ ). This hypothesis is corroborated by the observation that the character of the dissociation changes with peptide length. Fig. 3a shows that for the tripeptide 1a heterolytic cleavage of the LG-anion a at $229 \mathrm{u} / \mathrm{e}$ is the only observed dissociation channel. However, for all longer oligopeptides we find the additional channel involving the transfer of a proton, which results in the separation of a neutral leaving group LG-H from a negatively charged peptide (Fig. 3b and Scheme 1). While the hexapeptide 2a still shows partial heterolysis, the longer peptides $3 \mathbf{a}$ and $\mathbf{4 a}$ dissociate exclusively under proton transfer, with the fragment at $\mathrm{m} / \mathrm{z}=$ $(M-230) \mathrm{u} / \mathrm{e}$. The proton transfer reaction is always accompanied by the formation of a second fragment at $m / z=(M-246) \mathrm{u} / \mathrm{e}$.

In contrast to that we have never observed proton transfer in our collision induced dissociation experiments (Fig. S5, ESI $\dagger$ ). Instead, the CID spectrum of the hexa- and nonapeptide $2 \mathrm{a}$ and 3a yield about $5 \%$ of heterolytic cleavage at $300 \mathrm{~K}$, and the nonapeptide spectrum shows the appearance of some backbone fragments.

A systematic variation of the leaving group $\mathbf{a}, \mathbf{b}, \mathbf{c}, \mathbf{d}$ at the tripeptide $\mathbf{1}$, confirmed our design hypothesis that the electron withdrawing fluorine substituents stabilize the negative charge on the LG phenolates and enable heterolytic cleavage. We correlate the heterolytic cleavage efficiencies with density functional theory (DFT) calculations (ESI $\dagger$ ) to shed light on our experimental findings. Initial conformations used in DFT calculations are modeled in terms of chemical constitution and further locally relaxed using manually created conformations. Short ab initio 
molecular dynamics (AIMD) simulations at $300 \mathrm{~K}$ further helped us to explore the potential energy surface (PES) for candidates while the electronic potential is provided by DFT at the PBE0/ 3-21G level of theory. Several conformational candidates are further locally optimized at $0 \mathrm{~K}$ at the PBE0/Def2TZVP ${ }^{35,36}$ level and lowest energy conformations are used in the following calculations. The energetics of the photocleavage process is addressed by relating heterolytic bond dissociation energies $(\mathrm{BDE}),{ }^{37}$ vertical electron detachment energies (VDE), ${ }^{38}$ fragment yields and $\mathrm{p} K_{\mathrm{a}}$ values. Additionally, mean thermal energies are estimated from calculated vibrational spectra in the harmonic approximation (Table S1, ESI $\dagger$ ).

Even though the energy of a single $266 \mathrm{~nm}$ photon $(4.7 \mathrm{eV})$ is smaller than the BDE of $\mathbf{1 a}(6.9 \mathrm{eV})$, it adds to a mean thermal energy of $1.4 \mathrm{eV}$ at $300 \mathrm{~K}$ and thus to a total internal energy of $6.1 \mathrm{eV}$, which is close enough to the BDE for fragmentation to occur after some intra-molecular reorganization. At lower temperature, here at $60 \mathrm{~K}$, the total internal energy of $4.8 \mathrm{eV}$ is far below the BDE value. This is consistent with the observation in Fig. $2 \mathrm{~b}$ that at $60 \mathrm{~K}$ two or more photons are required in most cases. Electron detachment cannot be entirely excluded, given
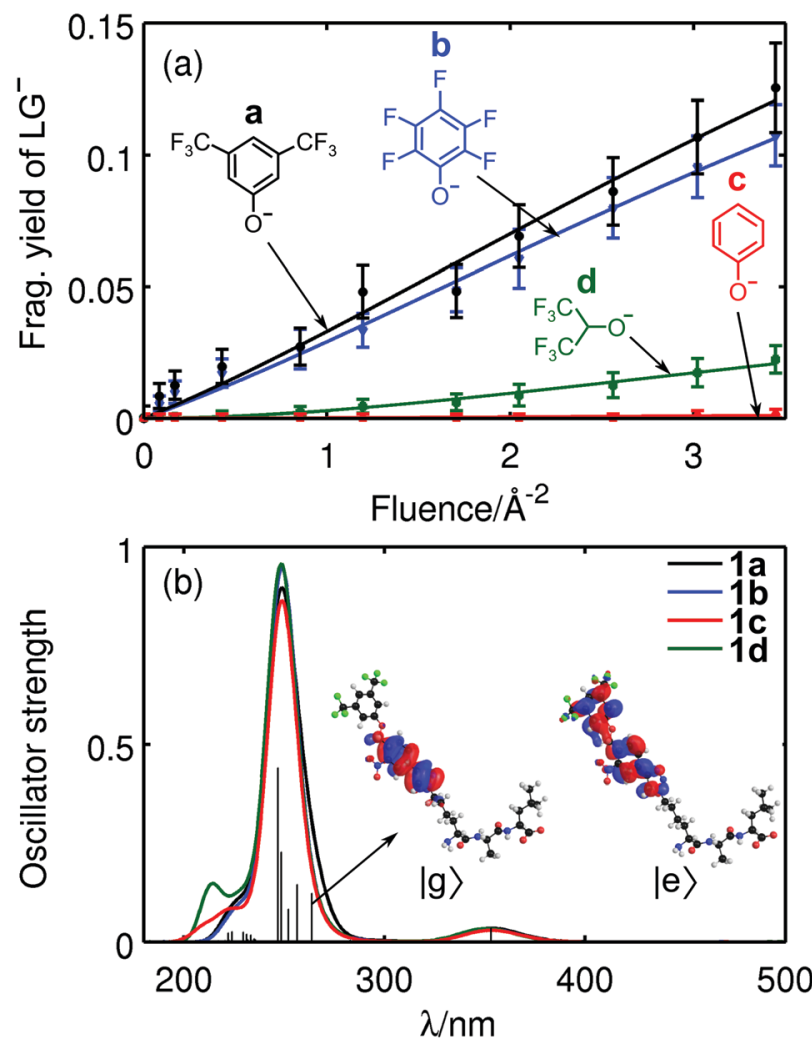

Fig. 4 (a) LG fragment yield of the functionalized tripeptide 1 as a function of the laser fluence. The four difference curves correspond to the same PCT core with four different leaving groups (a, b, c and $\mathbf{d}$ ). (b) The oscillator strength of the tripeptides $\mathbf{1 a - 1 d}$ is obtained by TDDFT. For simplicity we show a Gaussian convolutions to the line spectrum only for peptide 1a. The arrow points to the NTOs with the largest eigenvalue for the transition close to $266 \mathrm{~nm}$. For the particular LG a the calculations find efficient charge transfer from the absorbing PCT towards the LG in the transition from the ground state $|g\rangle$ to the excited state $|e\rangle$. the computed VDE values of $4.6 \mathrm{eV}$, especially since the experimental fragment collection efficiency is not exactly known.

Apparently, for some LGs heterolytic cleavage becomes less probable than a dissociation involving proton transfer (1c). Heterolytic cleavage must leave a zwitterionic peptide behind which might be favored by the formation of a tropylium cation. Preliminary DFT calculations (ESI $\dagger$ ) indicate that this structure is of comparable stability to the corresponding benzyl cation. It remains, however, an open task to model detailed reaction pathways and to evaluate the barriers for the intermediates. We also find that the trend in fragmentation yields for compounds 1a-1d (Fig. 4a), correlate with the $\mathrm{p} K_{\mathrm{a}}$ values of the protonated leaving groups LG-H (Table S1, ESI $\dagger$ ), even though the latter also include ion solvation energies.

To compare the optical response of the tripeptides 1 with different LGs, time-dependent density functional theory (TDDFT) calculations were performed at the same level of theory as before, involving 100 excited states. Gaussian convolutions to the calculated line spectra show strong absorption around $250 \mathrm{~nm}$ for 1a-1d (Fig. 4b). Electronic excitation analysis based on natural transition orbitals (NTO) ${ }^{39}$ confirms that the UV light excites the PCT rather than the peptide. We also find that the absorption spectra do not significantly change upon exchange of the LG.

The photocleavage of $o$-nitrobenzylethers and related nitroaryls in solution is well documented in the literature. ${ }^{40,41}$ Since cleavage of the 2-phenoxy-methyl-nitrobenzene can already be realized with electrons and atoms of the photolinker, that is without the involvement of solvent molecules, site-specific dissociation should also be possible in the gas phase, ${ }^{33}$ as seen in our experiments.

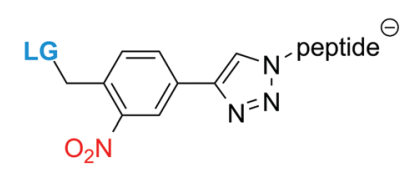

$$
\begin{aligned}
& \text { 1a } \text { peptide }=1, L G=a \\
& \text { 3a }
\end{aligned}
$$
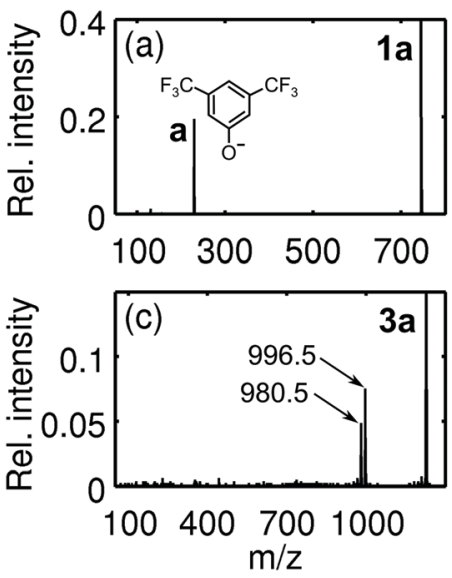
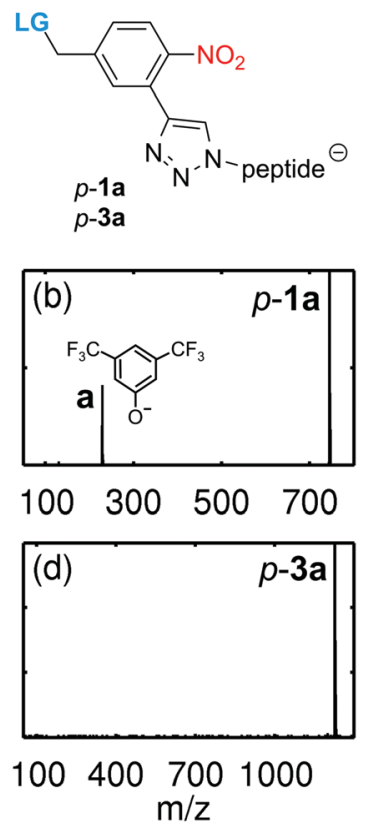

Fig. 5 Effect of nitro-group position on photocleavage efficiency. Heterolysis in the tripeptide is little affected by moving the nitro-group to the para-position of the LG ( $a$ and $b)$ whereas cleavage under proton transfer is suppressed in the nonapeptide (c and $d$ ). 
To decipher the role of the nitro group, isomers of the tripeptide 1a and the nonapeptide $\mathbf{3 a},(p-\mathbf{1 a}, p$-3a) were synthesized with the nitro-group in the para-position of the benzylic ether function (Fig. 5). We find that $p$-1a and 1a cleave with a comparable heterolytic efficiency, corroborating the thermal nature of the process. This is markedly different from the behavior in solution (DMSO- $\mathrm{d}_{6}$ ) where irradiation of $p$-1a at $254 \mathrm{~nm}$ does not yield any cleavage, while it does for 1a (Fig. S8, ESI $\dagger$ ). However, the modified nonapeptide $p$-3a does not cleave under conditions where $\mathbf{3 a}$ dissociates. This indicates that the proton transfer pathway resembles the accepted solution phase mechanism ${ }^{42}$ and can be suppressed by repositioning the nitro group. The heterolytic channel, on the other hand, is too slow for the para-functionalized nonapeptides. For the short peptides $\mathbf{1 a - 1} \mathbf{b}$, the sum of photon $(4.7 \mathrm{eV})$ and thermal energies $(\approx 1.3 \mathrm{eV})$ is sufficient to release a negatively charged LG, and the heterolytic mechanism is observed. For larger peptides, however, the heterolytic process seems less favourable and the proton transfer pathway takes over, which sensitively depends on the proximity of the $\mathrm{NO}_{2}$ to the leaving group.

\section{Conclusions}

Our results demonstrate that photocleavable peptides can efficiently and selectively be cleaved in the gas-phase using UV light. The tailored peptides undergo different dissociation mechanisms depending on the nature of the LG, the size of the peptides, the molecular temperature and the position of the nitro group within the PCT.

We have demonstrated that small peptides can undergo thermally assisted heterolytic photocleavage in the gas phase while longer oligopeptides follow a dissociation path presumably involving proton transfer to the LG, more closely resembling the solution phase mechanism. This insight can contribute to the design of new peptide labels for proteomics. ${ }^{19,43}$

Photoactive groups have recently been studied for applications in solution and optimized response to a desired wavelength. ${ }^{42,44}$ In our current work we have explored the influence of peptide size and the nature of the LG on the heterolytic photocleavage efficiency in the gas phase. Since our longest oligopeptides preferentially follow a PD mechanism with proton transfer, neutralization of large polypeptides or eventually proteins in the gas phase may require the charge to be stabilized on the leaving group. Our current experiments were targeting non-aromatic peptides where we can avoid an absorption competition between the tag and the aromatic chromophores. Future experiments will explore red-shifted tags, which will then be applicable to aromatic peptides, also.

Both the neutral and the charged dissociation pathways are interesting and useful for gaining optical control over the motional states of molecules. Photocleavage can be realized with high spatial control and very precise timing. This technique may be used for post-neutralizing singly charged anion beams, which have been previously guided and cooled in a buffer gas environment. The optically induced gas phase photo-depletion of the parent peak is also promising for realizing coherent beam splitters based on photo-depletion of a molecular beam with nanometer resolution. ${ }^{45}$ This will become important for quantum optics and metrology experiments with complex neutral biomolecules.

\section{Conflicts of interest}

There are no conflicts to declare.

\section{Acknowledgements}

This project has received funding from the European Research Council (ERC) under the European Union's Horizon 2020 research and innovation programme (Grant No. 320694), the Swiss National Science Foundation (200020-159730), and the Swiss Nanoscience Institute at the University of Basel (P1403). We are thankful to M. C. Böhm for computational advice and M. Kerschbaum for support in the ESI measurements. We thank N. Münch und D. Häussinger for NMR experiments. The computational results were obtained using the Vienna Scientific Cluster (VSC).

\section{Notes and references}

1 M. F. Jarrold, Annu. Rev. Phys. Chem., 2000, 51, 179-207.

2 M. Schennach and K. Breuker, Angew. Chem., Int. Ed., 2014, 53, 164-168.

3 O. S. Skinner, F. W. McLafferty and K. Breuker, J. Am. Soc. Mass Spectrom., 2012, 23, 1011-1014.

4 T. Meyer, V. Gabelica, H. Grubmüller and M. Orozco, Wiley Interdiscip. Rev.: Comput. Mol. Sci., 2013, 3, 408-425.

5 P. S. Walsh, K. N. Blodgett, C. McBurney, S. H. Gellman and T. S. Zwier, Angew. Chem., Int. Ed., 2016, 55, 14618-14622.

6 C. Baldauf and M. Rossi, J. Phys.: Condens. Matter, 2015, 27, 493002.

7 F. Bierau, P. Kupser, G. Meijer and G. von Helden, Phys. Rev. Lett., 2010, 105, 133402.

8 A. Ostendorf, C. B. Zhang, M. Wilson, D. Offenberg, B. Roth and S. Schiller, Phys. Rev. Lett., 2006, 97, 243005.

9 M. Guidi, U. J. Lorenz, G. Papadopoulos, O. V. Boyarkin and T. R. Rizzo, J. Phys. Chem. A, 2009, 113, 797-799.

10 J. M. Weber, I. N. Ioffe, K. M. Berndt, D. Löffler, J. Friedrich, O. T. Ehrler, A. S. Danell, J. H. Parks and M. M. Kappes, J. Am. Chem. Soc., 2004, 126, 8585-8589.

11 M. Vonderach, O. T. Ehrler, K. Matheis, P. Weis and M. M. Kappes, J. Am. Chem. Soc., 2012, 134, 7830-7841.

12 H. N. Chapman, P. Fromme, A. Barty, T. A. White, R. A. Kirian, A. Aquila, M. S. Hunter, J. Schulz, D. P. DePonte, U. Weierstall, R. B. Doak, F. R. N. C. Maia, A. V. Martin, I. Schlichting, L. Lomb, N. Coppola, R. L. Shoeman, S. W. Epp, R. Hartmann, D. Rolles, A. Rudenko, L. Foucar, N. Kimmel, G. Weidenspointner, P. Holl, M. Liang, M. Barthelmess, C. Caleman, S. Boutet, M. J. Bogan, J. Krzywinski, C. Bostedt, S. Bajt, L. Gumprecht, B. Rudek, B. Erk, C. Schmidt, A. Hömke, C. Reich, D. Pietschner, L. Strüder, G. Hauser, 
H. Gorke, J. Ullrich, S. Herrmann, G. Schaller, F. Schopper, H. Soltau, K. Kühnel, M. Messerschmidt, J. D. Bozek, S. P. Hau-Riege, M. Frank, C. Y. Hampton, R. G. Sierra, D. Starodub, G. J. Williams, J. Hajdu, N. Timneanu, M. M. Seibert, J. Andreasson, A. Rocker, O. Jönsson, M. Svenda, S. Stern, K. Nass, R. Andritschke, C. Schröter, F. Krasniqi, M. Bott, K. E. Schmidt, X. Wang, I. Grotjohann, J. M. Holton, T. R. M. Barends, R. Neutze, S. Marchesini, R. Fromme, S. Schorb, D. Rupp, M. Adolph, T. Gorkhover, I. Andersson, H. Hirsemann, G. Potdevin, H. Graafsma, B. Nilsson and J. C. H. Spence, Nature, 2011, 470, 73-77.

13 T. Gorkhover, S. Schorb, R. Coffee, M. Adolph, L. Foucar, D. Rupp, A. Aquila, J. D. Bozek, S. W. Epp, B. Erk, L. Gumprecht, L. Holmegaard, A. Hartmann, R. Hartmann, G. Hauser, P. Holl, A. Hömke, P. Johnsson, N. Kimmel, K. Kühnel, M. Messerschmidt, C. Reich, A. Rouzée, B. Rudek, C. Schmidt, J. Schulz, H. Soltau, S. Stern, G. Weidenspointner, B. White, J. Küpper, L. Strüder, I. Schlichting, J. Ullrich, D. Rolles, A. Rudenko, T. Möller and C. Bostedt, Nat. Photonics, 2016, 10, 93-97.

14 P. Schanen, D. Yang, R. Weinkauf and E. Schlag, Int. J. Mass Spectrom. Ion Processes, 1997, 167, 447-470.

15 J. L. Stephenson and S. A. McLuckey, J. Am. Chem. Soc., 1996, 118, 7390-7397.

16 X. Pan, P. Cloutier, D. Hunting and L. Sanche, Phys. Rev. Lett., 2003, 90, 208102.

17 L. Joly, R. Antoine, A.-R. Allouche, M. Broyer, J. Lemoine and P. Dugourd, J. Am. Chem. Soc., 2007, 129, 8428-8429.

18 J. A. Stearns, S. Mercier, C. Seaiby, M. Guidi, O. V. Boyarkin and T. R. Rizzo, J. Am. Chem. Soc., 2007, 129, 11814-11820.

19 J. S. Brodbelt, Chem. Soc. Rev., 2014, 43, 2757-2783.

20 R. Antoine and P. Dugourd, Phys. Chem. Chem. Phys., 2011, 13, 16494-16509.

21 M. Bouakil, A. Kulesza, S. Daly, L. MacAleese, R. Antoine and P. Dugourd, J. Am. Soc. Mass Spectrom., 2017, 28, 2181-2188.

22 A. R. Milosavljevic, C. Nicolas, J. Lemaire, C. Dehon, R. Thissen, J.-M. Bizau, M. Réfrégiers, L. Nahon and A. Giuliani, Phys. Chem. Chem. Phys., 2011, 13, 15432-15436.

23 W. D. Price, R. A. Jockusch and E. R. Williams, J. Am. Chem. Soc., 1998, 120, 3474-3484.

24 T. Ly and R. R. Julian, J. Am. Chem. Soc., 2008, 130, 351-358.

25 D. A. Polasky, F. Lermyte, M. Nshanian, F. Sobott, P. C. Andrews, J. Loo and B. T. Ruotolo, Anal. Chem., 2018, 90, 2756-2764.
26 R. Hodyss, H. A. Cox and J. Beauchamp, J. Am. Chem. Soc., 2005, 127, 12436-12437.

27 T. Juffmann, H. Ulbricht and M. Arndt, Rep. Prog. Phys., 2013, 76, 086402.

28 L. Mairhofer, S. Eibenberger, J. P. Cotter, M. Romirer, A. Shayeghi and M. Arndt, Angew. Chem., Int. Ed., 2017, 56, 10947.

29 S. Eibenberger, S. Gerlich, M. Arndt, J. Tüxen and M. Mayor, New J. Phys., 2011, 13, 043033.

30 J. Tüxen, S. Gerlich, S. Eibenberger, M. Arndt and M. Mayor, Chem. Commun., 2010, 46, 4145-4147.

31 J. Rodewald, P. Haslinger, N. Dörre, B. A. Stickler, A. Shayeghi, K. Hornberger and M. Arndt, Appl. Phys. B: Lasers Opt., 2017, 123, 3.

32 J. B. Fenn, M. Mann, C. K. Meng, S. F. Wong and C. M. Whitehouse, Mass Spectrom. Rev., 1990, 9, 37-70.

33 U. Sezer, P. Geyer, M. Kriegleder, M. Debiossac, A. Shayeghi, M. Arndt, L. Felix and M. Mayor, Beilstein J. Nanotechnol., 2017, 8, 325.

34 J. Friedrich, S. Gilb, O. T. Ehrler, A. Behrendt and M. M. Kappes, J. Chem. Phys., 2002, 117, 2635-2644.

35 C. Adamo and V. Barone, J. Chem. Phys., 1999, 110, 6158-6170.

36 F. Weigend and R. Ahlrichs, Phys. Chem. Chem. Phys., 2005, 7, 3297-3305.

37 S. J. Blanksby and G. B. Ellison, Acc. Chem. Res., 2003, 36, 255-263.

38 J. M. Herbert and M. Head-Gordon, J. Phys. Chem. A, 2005, 109, 5217-5229.

39 R. L. Martin, J. Chem. Phys., 2003, 118, 4775-4777.

40 P. Sebej, T. Solomek, L. Hroudna, P. Brancova and P. Klan, J. Org. Chem., 2009, 74, 8647-8658.

41 T. Šolomek, S. Mercier, T. Bally and C. G. Bochet, Photochem. Photobiol. Sci., 2012, 11, 548-555.

42 P. Klán, T. Šolomek, C. G. Bochet, A. Blanc, R. Givens, M. Rubina, V. Popik, A. Kostikov and J. Wirz, Chem. Rev., 2012, 113, 119-191.

43 Q. Enjalbert, M. Girod, R. Simon, J. Jeudy, F. Chirot, A. Salvador, R. Antoine, P. Dugourd and J. Lemoine, Anal. Bioanal. Chem., 2013, 405, 2321-2331.

44 M. J. Hansen, W. A. Velema, M. M. Lerch, W. Szymanski and B. L. Feringa, Chem. Soc. Rev., 2015, 44, 3358-3377.

45 N. Dörre, J. Rodewald, P. Geyer, B. von Issendorff, P. Haslinger and M. Arndt, Phys. Rev. Lett., 2014, 113, 233001. 